\title{
INFLUÊNCIA DA VARIABILIDADE ESPACIAL DE ATRIBUTOS QUÍMICOS DE UM LATOSSOLO NA APLICAÇÃO DE INSUMOS PARA CULTURA DE CANA-DE-AÇÚCAR
}

\author{
Influence of the spatial variability of latosol chemical attributes \\ and input application for sugarcane culture
}

\author{
Zigomar Menezes de Souza ${ }^{1}$, Diogo Mazza Barbieri², José Marques Júnior³, Gener Tadeu Pereira ${ }^{4}$, \\ Milton César Costa Campos ${ }^{2}$
}

\begin{abstract}
RESUMO
As técnicas de aplicação localizada e em taxas variadas de insumos agrícolas com auxílio da geoestatística têm sido umas das áreas de concentração de esforços recentes em pesquisas e desenvolvimento ligado à agricultura de precisão. Portanto, o trabalho teve como objetivo avaliar a necessidade de aplicação de calcário e fósforo com base no comportamento espacial de atributos químicos do solo. Foram feitas amostragens do solo a intervalos regulares de $50 \mathrm{~m}$, em uma área de $42 \mathrm{ha}$, na profundidade de $0,0-0,2 \mathrm{~m}$ para determinação do $\mathrm{pH}, \mathrm{Ca}, \mathrm{Mg}, \mathrm{P}, \mathrm{V} \%$ e cálculo da necessidade de calagem e superfosfato triplo. Os dados foram submetidos às análises da estatística descritiva, geoestatística e interpolação por krigagem. O uso da técnica de geoestatística possibilitou uma precisa descrição dos atributos do solo o que permitiu a definição de zonas de manejo, indicando os locais de ocorrência de déficit e excesso. A variabilidade espacial dos atributos químicos do solo, possibilita a recomendação de doses de calcário e fósforo com taxas variadas, proporcionando economia e maior eficiência na aplicação.
\end{abstract}

Termos para indexação: Geoestatística, manejo localizado, calagem.

\begin{abstract}
Geostatistics and precision agriculture supports on techniques for local application of agricultural amendments at varied rates, has been one of the areas where recent efforts of research and development have been focused. Therefore, the present paper aimed to evaluate lime and phosphorus application needs according to the spatial distribution of soil chemical attributes. Soil samples were collected from 0.0-0.20 m soil layer in the intersection points of a 42 ha grid, $50 \mathrm{~m}$ distant from each other. $\mathrm{Ca}, \mathrm{Mg}, \mathrm{P}, \mathrm{pH}, \mathrm{V} \%$ as well as P and liming needs were determined. The data set was submitted to descriptive analysis, geostatistics and kriging analysis. The geostatistics analysis allowed a precise description of soil attributes and, consequently, the definition of site-specific management indicating where nutrient deficit or excess occurred. The spatial variability of soil chemical attributes allowed the recommendation of lime and phosphorus at suitable rates, providing economy and greater efficiency in their application.
\end{abstract}

Index terms: Geostatistics, site-specific management, lime.

\section{(Recebido em 4 de novembro de 2005 e aprovado em 14 de agosto de 2006)}

\section{INTRODUÇÃO}

O alto dinamismo do sistema agrícola no espaço e no tempo, influenciado por fatores inerentes à planta, ao solo, ao clima e às ações antrópicas e a globalização da economia têm levado a uma necessidade de otimização deste sistema. Uma destas otimizações, associada ao conceito de Agricultura de Precisão, está relacionada à aplicação de agrotóxicos e fertilizantes que quando aplicados de maneira ineficiente geram problemas ambientais e aumento do custo de produção (DODERMANN \& PING, 2004). De acordo com Mapa \&
Kumaragamage (1996), o termo Agricultura de Precisão é o conjunto de técnicas que visam otimizar a produção com o menor impacto ambiental possível.

A solução hoje utilizada é a de enfocar grandes áreas e entendê-las como homogêneas, levando ao conceito da necessidade média para a aplicação dos insumos o que faz com que, por exemplo, a mesma formulação e/ou quantidade do fertilizante seja utilizada para toda a área, atendendo apenas as necessidades médias e não considerando, desta forma, as necessidades específicas de cada parte do campo (TSCHIEDEL \& FERREIRA, 2002).

\footnotetext{
'Engenheiro Agrônomo, Professor Dr. da Faculdade de Engenharia Agrícola - UNICAMP - Cidade Universitária Zeferino Vaz, s/n - Cx. P. 6011 13083-875 - Campinas, SP -zigomarms@ agr.unicamp.br

2Engenheiros Agrônomos, Mestrandos da Faculdade de Ciências Agrárias e Veterinárias - UNESP - Via de Acesso Professor Paulo Donato Castellane, s/n - 14884-900 - Jaboticabal, SP - diogombarbieri@ yahoo.com.br; mcesar@fcav.unesp.br

${ }^{3}$ Engenheiro Agrônomo, Professor Dr. da Faculdade de Ciências Agrárias e Veterinárias, Departamento de Solos e Adubos - UNESP - Via de Acesso Prof. Paulo Donato Castellane, s/n - 14884-900 - Jaboticabal, SP - marques @ fcav.unesp.br

${ }^{4}$ Estatístico, Professor Dr. da Faculdade de Ciências Agrárias e Veterinárias - Departamento de Ciências Exatas - UNESP - Via de Acesso Prof. Paulo Donato Castellane, s/n - 14884-900 - Jaboticabal, SP - genertp@fcav.unesp.br
} 
As técnicas estatísticas empregadas na ciência agrícola tradicionalmente pressupõe estacionaridade e independência dos dados. Atributos do solo no entanto, podem apresentar dependência espacial em escalas que variam da ordem de poucos metros a alguns quilômetros (WHITE et al., 1997; YANG et al., 2001). Além disso, é importante constatar que o solo é resultado da ação de alguns fatores que atuam em larga escala e cujo efeito é alterado por fatores que atuam em escalas menores e em diferentes níveis de superposição de efeitos o que proporciona um caráter hierárquico para a estrutura de variação das propriedades no espaço (GOOVAERTS, 1997).

De acordo com Cerri (2001), pode-se sugerir que a quantificação da variabilidade espacial é útil por dois motivos. O primeiro é que o conhecimento dos erros associados ao modelo permite avaliar a qualidade dos resultados definindo se estes atendem ao esperado, e se a qualidade é superior à necessária. Por outro lado, a qualidade pode ser inferior à necessária. Neste caso, talvez seja necessário coletar mais dados, calibrar melhor o modelo ou até mesmo escolher melhor um método de predição. $\mathrm{O}$ segundo é no mapeamento dos atributos em questão que, para a agricultura de precisão, é a base para gerar mapas de aplicação de insumos à taxa variável.

Este conceito é evidenciado pelo trabalho de Borgelt et al. (1994), o qual depois de amostrar o pH do solo de uma área de 8,8 hectares, concluíram que sem a utilização da técnica de aplicação localizada, 9 a $12 \%$ dessa área receberia uma quantidade de calcário acima da recomendada e 37 a 41 $\%$ receberia uma quantidade abaixo da recomendada.

O presente trabalho teve como objetivo avaliar a necessidade de aplicação de calcário e fósforo baseado no comportamento espacial de atributos químicos de um latossolo sob o cultivo de cana-de-açúcar na região de Jaboticabal, SP.

\section{MATERIAL E MÉTODOS}

A área de estudo localiza-se na fazenda Santa Bárbara município de Guariba-SP, região de Jaboticabal, nordeste do Estado de São Paulo, a qual está inserida na província geomórfica das Cuestas Basálticas, limite com o Planalto Ocidental Paulista. A região apresenta relevo suave ondulado com altitude média de $600 \mathrm{~m}$. As coordenadas geográficas são de $21^{\circ} 19^{\prime}$ a $21^{\circ} 20^{\prime}$ S e $48^{\circ} 13^{\prime}$ a $48^{\circ} 14^{\prime}$ 'WGr. O clima da região foi classificado segundo Köppen, como subtropical com inverno seco (Cwa), com precipitação média de $1.400 \mathrm{~mm}$, temperatura média anual de $21^{\circ} \mathrm{C}$, com temperatura no mês mais quente superior a $22{ }^{\circ} \mathrm{C}$ e a do mês mais frio inferior a $18^{\circ} \mathrm{C}$.
O material de origem dos solos regionais é resultado do intemperismo e retrabalhamento do basalto, formação Serra Geral, Grupo São Bento, arenitos do Grupo Bauru e depósitos de aluviões. O solo da área em estudo foi classificado como Latossolo Vermelho eutroférrico textura muito argilosa (LVef), segundo Embrapa (1999).

O cultivo intensivo de cana-de-açúcar com queima antes da colheita, vem sendo conduzido por um período de mais de 30 anos consecutivos. A área foi renovada no ano de 1995, com adubação de $600 \mathrm{~kg} \mathrm{ha}^{-1}$ da fórmula 5-2525 e nos anos de 1996, 1997 e 1998 a adubação foi de $500 \mathrm{~kg} \mathrm{ha}^{-1} \mathrm{da}$ mesma formulação. Nos anos de 1999 e 2000 a área não recebeu adubação química, apenas foi aplicado $80 \mathrm{~m}^{3} \mathrm{ha}^{-1}$ de vinhaça como adubação orgânica. Para o cultivo da área, foi realizada uma escarificação e uma gradagem leve (destorroamento).

Os solos foram amostrados nos pontos de cruzamento de uma malha, com intervalos regulares de 50 $\mathrm{m}$, perfazendo um total de 206 pontos em uma área de 42 ha. A malha foi montada em julho de 2001, utilizando um equipamento topográfico (teodolito) para o estaqueamento. Após o estaqueamento foi efetuado o georreferenciamento de cada ponto da malha com um equipamento de GPS.

A coleta do solo foi realizada com trado tipo holandês de 0,05 $\mathrm{m}$ de diâmetro na profundidade de 0,0-0,2 $\mathrm{m}$ em 206 pontos. As amostras coletadas foram levadas ao laboratório e submetidos à análise química. $\mathrm{O} \mathrm{pH}$ foi determinado potenciometricamente utilizando-se relação 1:2,5 (solo: $\mathrm{CaCl}_{2} 0,01 \mathrm{~mol} \mathrm{~L}^{-1}$ ). O cálcio, magnésio e potássio trocáveis e, o fósforo disponível foram extraídos utilizandose o método da resina trocadora de íons (RAIJ et al., 2001).

Para efeito de comparação foram realizados dois diferentes tipos de cálculos para a necessidade de calagem e para adubação com P na área em estudo. Primeiramente, os cálculos foram feitos considerando-se que a área fosse homogênea e posteriormente considerou a variabilidade espacial da área para a realização do cálculo.

Para o cálculo da necessidade de corretivo utilizouse a fórmula proposta por Raij et al. (1997), na qual leva-se em consideração o nível de acidez atual do solo, a CTC e o nível de saturação por bases ideal da cultura, que no caso da cana-de-açúcar é de $60 \%$. Para efeito de cálculo o PRNT do calcário foi considerado $100 \%$. Para o cálculo da adubação de $\mathrm{P}$ foi utilizada a tabela de adubação proposta por Raij et al. (1997) e, a produtividade esperada considerada foi de 100 a $150 \mathrm{t} \mathrm{ha}^{-1}$.

Os atributos químicos do solo foram submetidos à análise estatística descritiva obtendo-se média, mediana, 
coeficiente de variação, assimetria e curtose e, tipo de distribuição dos dados, com o uso do pacote estatísticos Sas (SCHLOTZHAVER \& LITTELL, 1997). Posteriormente, foram realizadas análises geoestatísticas por meio da construção de semivariogramas com o auxílio do programa GS+ (ROBERTSON, 1998) e confecção dos mapas de krigagem através do programa SURFER (1999). A análise do grau de dependência espacial dos atributos foi realizada segundo Cambardella et al. (1994), em que são considerados de dependência espacial forte os semivariogramas que têm um efeito pepita menor ou igual $25 \%$ do patamar, moderada entre $25 \%$ e $75 \%$, e fraca quando for maior que $75 \%$.

\section{RESULTADOS E DISCUSSÃO}

Os resultados da estatística descritiva para os atributos químicos são apresentados na Tabela 1. Os resultados referentes ao teste Kolmogorov-Smirnov indicaram normalidade para o V\%. De acordo com Isaaks \& Srivastava (1989), o coeficiente de assimetria é mais sensível a valores extremos do que a média e o desvio padrão, uma vez que um único valor pode influenciar fortemente o coeficiente de assimetria, pois os desvios entre cada valor e a média são elevados à terceira potência. Para o presente trabalho foram encontrados valores próximos a zero para todos os atributos estudados (Tabela 1).

Isto demonstra que todos os atributos envolvidos no estudo estão aproximando-se de uma distribuição normal indicando que os dados estão adequados para o uso da geoestatística. Outra indicação de normalidade é que os valores de média e mediana, para todas as variáveis, estão próximos, mostrando distribuições simétricas (Tabela 1). Mais importante que a normalidade é que os semivariogramas apresentem patamares bem definidos e, que a distribuição não apresente caudas muito alongadas, o que poderia comprometer as estimativas da krigagem, as quais são baseadas nos valores médios (ISAAKS \& SRIVASTAVA, 1989).

De acordo com a classificação de Warrick \& Nielsen (1980), valores de coeficiente de variação baixo foram encontrados para o $\mathrm{pH}$. Para a variável V\% foram encontrados valores médios e para os restantes dos atributos foram encontrados valores altos para o coeficiente de variação (Tabela 1). Souza (2004), estudando a variabilidade espacial de atributos químicos na cultura de cana-de-açúcar, também encontrou valor de CV baixo para o $\mathrm{pH}$ e altos para as variáveis $\mathrm{P}, \mathrm{Ca}$ e $\mathrm{Mg}$.

Todos os atributos estudados apresentaram dependência espacial, a qual é expressa por meio dos ajustes aos modelos de semivariogramas (Tabela 2 e Figura 1). O modelo esférico ajustou-se aos dados de todas as variáveis estudadas com exceção da variável V\%, o qual ajustou-se ao modelo exponencial. Segundo Grego \& Vieira (2005), o modelo matemático esférico é o que predomina nos trabalhos em ciência do solo. Mcbratney \& Webster (1986) estudaram modelos de ajuste do semivariograma para os atributos do solo e relataram que os modelos esférico e exponencial são os mais encontrados.

O alcance fornece informações importantes para planejamento e avaliação experimental, além de subsidiar informações sobre a gênese e evolução dos solos na paisagem e, limites entre classes taxonômicas de solos. Os atributos em estudo apresentaram dois grupos de valores próximos de alcance, valores de alcance variando de 350 a $650 \mathrm{~m}$ (pH, Ca, Mg e P) e de 100 a 290 (V\%, necessidade de calagem e superfosfato triplo), evidenciando uma menor continuidade estrutural para as variáveis $\mathrm{V} \%$, necessidade de calagem e superfosfato triplo (Tabela 2).

TABELA 1 - Estatística descritiva para os atributos químicos e necessidade de calagem e superfosfato triplo.

\begin{tabular}{|c|c|c|c|c|c|c|}
\hline Atributo & Média & Mediana & $\mathbf{C V}$ & Assimetria & Curtose & $\mathbf{1}$ \\
\hline $\mathrm{pH}$ & 5,2 & 5,2 & 6,4 & 0,3 & $-0,3$ & 0,09 \\
\hline Cálcio $\left(\mathrm{mmol}_{\mathrm{c}} \mathrm{dm}^{-3}\right)$ & 37,6 & 37,0 & 25,6 & 0,7 & $-0,3$ & 0,09 \\
\hline Magnésio $\left(\mathrm{mmol}_{\mathrm{c}} \mathrm{dm}^{-3}\right)$ & 12,3 & 12,0 & 25,0 & 0,3 & $-0,5$ & 0,10 \\
\hline Fósforo $\left(\mathrm{mg} \mathrm{dm}^{-3}\right)$ & 26,4 & 26,0 & 32,1 & 0,4 & $-0,6$ & 0,09 \\
\hline $\mathrm{V} \%$ & 59,3 & 59,8 & 16,9 & $-0,03$ & $-0,8$ & $0,06^{\mathrm{ns}}$ \\
\hline Necessidade de calagem $\left(\mathrm{t} \mathrm{ha}^{-1}\right)$ & 1,10 & 1,14 & 43,2 & 0,5 & 0,4 & 0,17 \\
\hline $\begin{array}{l}\text { Necessidade de superfosfato } \\
\text { triplo }\left(\mathrm{kg} \mathrm{ha}^{-1}\right)\end{array}$ & 214,5 & 211,0 & 35,1 & 0,2 & 0,9 & 0,2 \\
\hline
\end{tabular}

${ }^{1} \mathrm{~d}=$ teste de normalidade, ${ }^{\mathrm{ns}}$ não-significativo pelo teste de Kolmogorov-Smirnov. 
TABELA 2 - Coeficientes e modelos dos semivariogramas ajustados para os atributos químicos, necessidade de calagem e superfosfato triplo.

\begin{tabular}{lccccc}
\hline Atributos & Modelo & $\begin{array}{c}\text { Efeito pepita } \\
\left(\mathbf{C}_{\mathbf{0}}\right)\end{array}$ & $\begin{array}{c}\text { Patamar } \\
\left(\mathbf{C}_{\mathbf{0}}+\mathbf{C}_{\mathbf{1}}\right)\end{array}$ & $\begin{array}{c}\text { Alcance } \\
(\mathbf{a})\end{array}$ & $\mathbf{C}_{\mathbf{0}} /\left(\mathbf{C}_{\mathbf{0}}+\mathbf{C}_{\mathbf{1}}\right)$ \\
\hline $\mathrm{pH}$ & Esférico & 0,04 & 0,09 & 345 & 44 \\
Cálcio $\left(\mathrm{mmol}_{\mathrm{c}} \mathrm{dm}^{-3}\right)$ & Esférico & 35,3 & 109,0 & 510 & 32 \\
Magnésio $\left(\mathrm{mmol}_{\mathrm{c}} \mathrm{dm}^{-3}\right)$ & Esférico & 4,9 & 9,9 & 473 & 50 \\
Fósforo $\left(\mathrm{mg} \mathrm{dm}^{-3}\right)$ & Esférico & 17,8 & 49,1 & 633 & 36 \\
V\% & Exponencial & 31,4 & 111,6 & 235 & 28 \\
Necessidade de calagem $\left(\mathrm{t} \mathrm{ha}^{-1}\right)$ & Esférico & 0,31 & 1,18 & 290 & 26 \\
Necessidade de superfosfato & Esférico & 381 & 1835 & 100 & 21 \\
triplo $\left(\mathrm{kg} \mathrm{ha}^{-1}\right)$ & & & & & \\
\hline
\end{tabular}

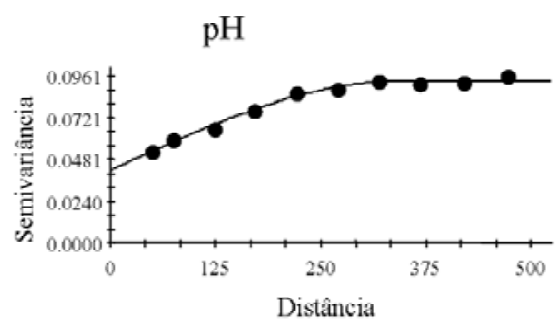

(m)

Fsf $(0,04 ; 0,09 ; 345)$

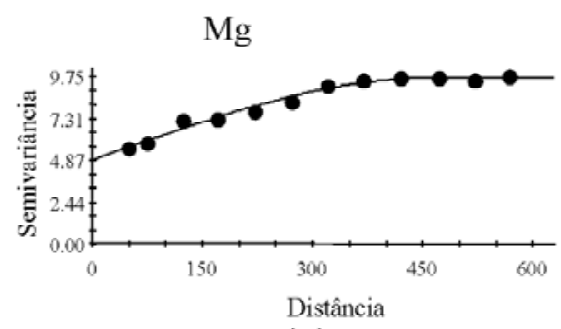

(m)

$\operatorname{Esf}(4,86 ; 9,9 ; 473)$

Necessidade de calagem (tha)

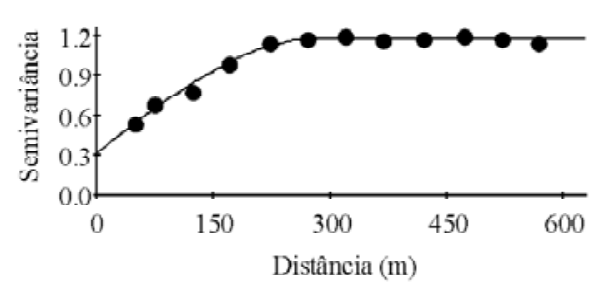

Esf (0,31-1,18-290)

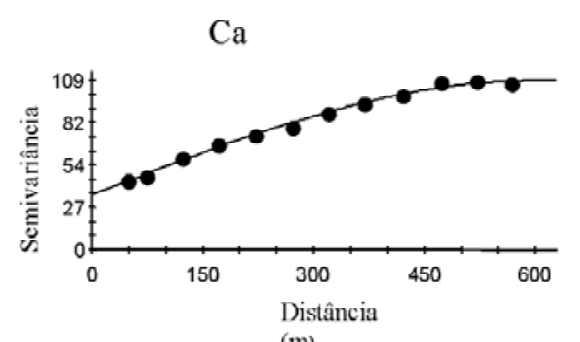

(m)

$\operatorname{Esf}(35,3 ; 109,0 ; 510)$

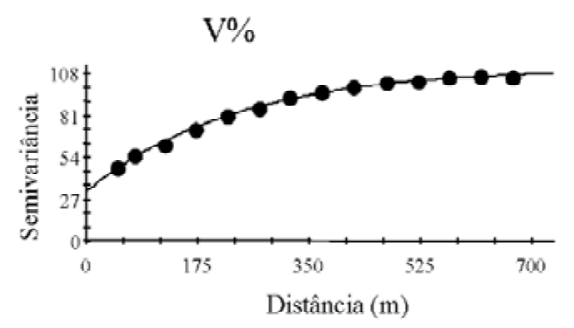

$\operatorname{Exp}(31,4 ; 111,6 ; 235)$

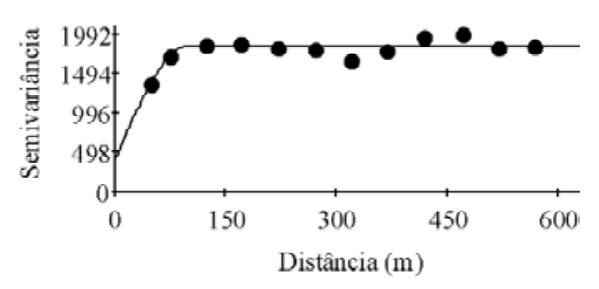

Esf (381-1835-100)

FIGURA 1 - Semivariogramas das variáveis $\mathrm{pH}$, cálcio $\left(\mathrm{mmol}_{\mathrm{c}} \mathrm{dm}^{-3}\right)$, magnésio $\left(\mathrm{mmol}_{\mathrm{c}} \mathrm{dm}^{-3}\right)$ e saturação por bases $(\mathrm{V} \%)$ na profundidade de 0,0-0,2 m e necessidade de calagem e superfosfato triplo. Esf e Exp $\left(\mathrm{C}_{0} ; \mathrm{C}_{0}+\mathrm{C}_{1} ; \mathrm{a}\right)$ é o modelo esférico e exponencial ajustado, $\mathrm{C}_{\mathrm{o}}=$ efeito pepita; $\mathrm{C}_{0}+\mathrm{C}_{1}=$ patamar; $\mathrm{a}=$ alcance. 
Todos os valores de alcance obtidos foram maiores que o valor de espaçamento entre as amostragens, indicando que as amostras estão correlacionadas umas a outras, o que permite que se façam interpolações (VIEIRA, 2000). A krigagem é uma técnica de estimação de locais não amostrados, usando propriedades estruturais do semivariograma confeccionados a partir de locais amostrados. Mapas de krigagem podem ser elaborados para aqueles atributos que apresentaram dependência espacial e, esta informação é usada para visualizar e melhor entender o padrão de distribuição espacial, além de definir diferentes zonas de manejo em uma determinada área.

Os parâmetros dos modelos de semivariogramas ajustados foram utilizados para estimar valores em locais não amostrados através da krigagem (Figura 2). Verificase por meio dos mapas de krigagem, para os atributos $\mathrm{pH}$, $\mathrm{Ca}, \mathrm{Mg}$ e $\mathrm{V} \%$, uma semelhança nos padrões de ocorrência mostrando que as variáveis apresentam correlação espacial na área em estudo. O mapa espacial da necessidade de calagem tem comportamento inverso das demais variáveis, uma vez que, onde ocorrem os maiores teores de $\mathrm{pH}, \mathrm{Ca}, \mathrm{Mg}$ e V\% a necessidade de calagem é menor.
$\mathrm{pH}$

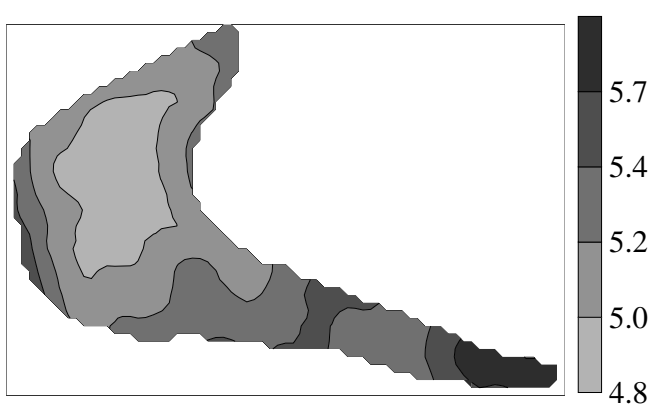

$\mathrm{Mg}$

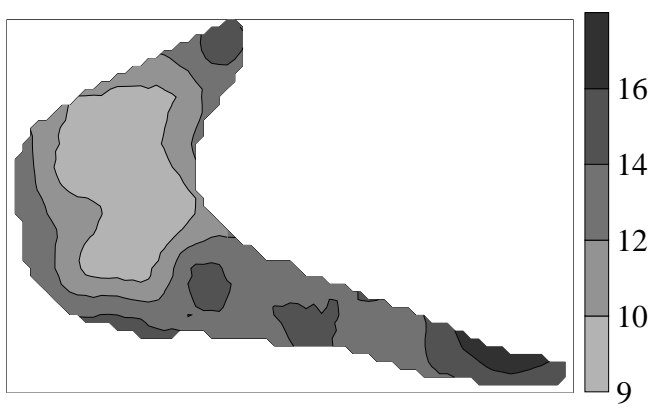

Necessidade de Calagem

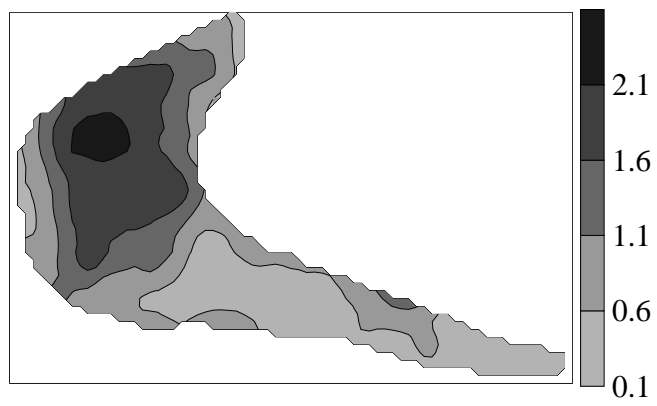

$\mathrm{Ca}$
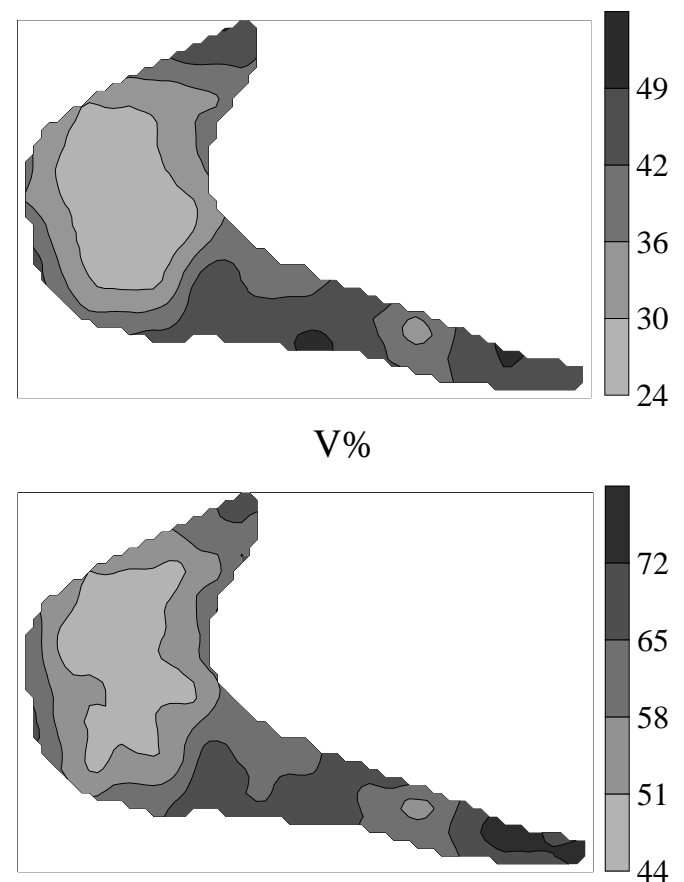

Necessidade de superfosfato triplo

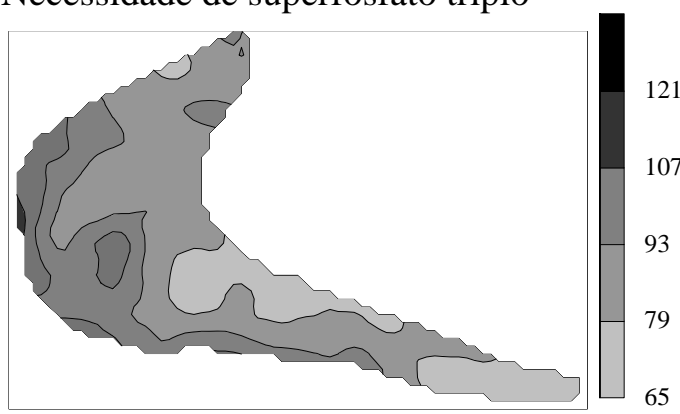

FIGURA 2 - Mapas de krigagem para o $\mathrm{pH}, \mathrm{Ca}\left(\mathrm{mmol}_{\mathrm{c}} \mathrm{dm}^{-3}\right), \mathrm{Mg}\left(\mathrm{mmol}_{\mathrm{c}} \mathrm{dm}^{-3}\right)$, saturação por bases $(\%)$, necessidade de calagem $\left(\mathrm{t} \mathrm{ha}^{-1}\right)$ e superfosfato triplo $\left(\mathrm{kg} \mathrm{ha}^{-1}\right)$. 
A necessidade de calagem obtida por meio da krigagem (Figura 2) variou de 0,0 a 2,1 t ha-1, para a aplicação de calcário a taxas variáveis. No entanto, utilizando-se a krigagem média seria necessário aplicar ao solo 1,10 t ha-1 de corretivo (PRNT igual a $100 \%$ ). O cálculo da necessidade de calagem, através do método convencional sem considerar a variabilidade espacial de atributos da acidez do solo, indicou ser necessário aplicar $1,50 \mathrm{t} \mathrm{ha}^{-1}$ de calcário.

Caso optasse pela aplicação de 1,50 t ha- ${ }^{-1}, 72 \%$ da área estudada receberia dose de calcário acima da desejada, ao passo que $28 \%$ da área não teria a acidez do solo corrigida para o nível ideal indicado para a cultura da canade-açúcar. Verifica-se que a aplicação do calcário por meio de taxa variada (Figura 2), possibilitaria uma grande economia, pois 30 ha não necessita da aplicação de calcário. A eficiência da correção da acidez do solo por meio de análise geoestatística também foi verificada por Borgelt et al. (1994).

Para o atributo $P$, percebe-se que as doses recomendadas para a aplicação de superfosfato triplo na área em estudo variam de 65 a $120 \mathrm{~kg} \mathrm{ha}^{-1}$ quando levado em conta as particularidades de cada célula da área (Figura 2). Caso a área seja considerada homogênea como é feito na agricultura tradicional, a dose aplicada na área seria de $120 \mathrm{~kg} \mathrm{ha}^{-1}$.

Analisando-se o mapa de $\mathrm{P}$, percebe-se que $20 \%$ da área necessita de apenas $80 \mathrm{~kg} \mathrm{ha}^{-1}$ de superfosfato triplo o que levaria a uma grande economia no fertilizante, proporcionando assim menores custos na compra do produto, menor contaminação ambiental, além de evitar uma superdosagem de fósforo na área.

Em um estudo desenvolvido por Corá et al. (2004) a avaliação da variabilidade espacial de atributos químicos na cultura de cana-de-açúcar, possibilitou definir zonas homogêneas de manejo específico, com o objetivo de efetuar a aplicação de calcário e fertilizantes a taxas variáveis, proporcionando, desta forma, um manejo mais eficiente e econômico da cultura.

\section{CONCLUSÕES}

O uso da técnica de geoestatística possibilitou uma precisa descrição dos atributos do solo o que permitiu a definição de zonas específicas de manejo.

A variabilidade espacial dos atributos químicos do solo possibilita a recomendação de doses de calcário e fósforo com taxas variadas, proporcionando economia e maior eficiência na aplicação.

\section{REFERÊNCIAS BIBLIOGRÁFICAS}

BORGELT, S. C.; SEARCY, S. W.; STOUT, B. A.; MULLA, D. J. Spatially variable liming rates: a method for determination. Transactions of the ASAE, Saint Joseph, v. 37, n. 5, p. 1499-1507, 1994.

CAMBARDELLA, C. A.; MOORMAN, T. B.; NOVAK, J. M.; PARKIN, T. B.; KARLEN, D. L.; TURCO, R. F.; KONOPKA, A. E. Field-scale variability of soil properties in Central Iowa. Soil Science Society of American Journal, Madison, v. 58, n. 5, p. 1501-1511, 1994.

CERRI, D. G. P. Desenvolvimento de um sistema de aplicação localizada de calcário a taxas variáveis. 2001. 90 f. Dissertação (Mestrado em Agronomia) - Escola Superior de Agricultura Luís de Queiroz, Universidade de São Paulo, Piracicaba, 2001.

CORÁ, J. E.; ARAUJO, A. V.; PEREIRA, G. T.; BERALDO, J. M. G. Variabilidade espacial de atributos do solo para adoção do sistema de agricultura de precisão na cultura de cana-de-açúcar. Revista Brasileira de Ciência do Solo, Viçosa, v. 28, n. 6, p. 1013-1021, 2004.

DODERMANN, A.; PING, J. L. Geostatistical integration of yield monitor data and remote sensing improves yield maps. Agronomy Journal, Madison, v. 96, n. 1, p. 285-297, 2004.

EMPRESA BRASILEIRA DE PESQUISA AGROPECUÁRIA. Centro Nacional de Pesquisa de Solos. Sistema brasileiro de classificação de solos. Brasília, DF, 1999. $412 \mathrm{p}$.

GOOVAERTS, P. Geoestatistics for natural resources evaluation. New York: Oxford University, 1997. 483 p.

GREGO, C. R.; VIEIRA, S. R. Variabilidade espacial de propriedades físicas do solo em uma parcela experimental. Revista Brasileira de Ciência do Solo, Viçosa, v. 29, n. 2, p. 169-177, 2005.

ISAAKS, E. H.; SRIVASTAVA, R. M. An introduction to applied geoestatistics. New York: Oxford University, 1989. $561 \mathrm{p}$.

MAPA, R. B.; KUMARAGAMAGE, D. Variability of soil properties in a tropical alfisol used for shifting cultivation. Soil Technology, Amsterdam, v. 9, n. 3, p. 187-197, 1996.

Ciênc. agrotec., Lavras, v. 31, n. 2, p. 371-377, mar./abr., 2007 
McBRATNEY, A. B.; WEBSTER, R. Choosing functions for semi-variograms of soil properties and fitting them to sampling estimates. Journal of Soil Science, Oxford, v. 37, n. 3, p. 617-639, 1986.

RAIJ, B. van; CANTARELLA, H.; QUAGGIO, J. A.; FURLANI, A. M. C. Recomendações de adubação e calagem para o estado de São Paulo. 2. ed. Campinas: Instituto Agronômico/Fundação IAC, 1997. 285 p. (Boletim técnico, 100).

RAIJ, B. van; ANDRADE, J. C.; CANTARELLA, H.; QUAGGIO, J. A. (Eds.). Análise química para avaliação da fertilidade de solos tropicais. Campinas: Instituto Agronômico, 2001. 285 p.

ROBERTSON, G. P. GS ${ }^{+}$geostatistics for the environmental sciences. Version 5.1. [S.1.]: Gamma Design Soffware, 1998. $152 \mathrm{p}$.

SCHLOTZHAVER, S. D.; LITTELL, R. C. SAS: system for elementary statistical analysis. 2. ed. Cory: SAS Institute, 1997. 905 p.

SOUZA, Z. M. Variabilidade espacial e atributos de um latossolo sob diferentes formas do relevo. 2004. $153 \mathrm{f}$. Tese (Doutorado em Produção Vegetal) - Faculdade de Ciências Agrárias e Veterinárias de Jaboticabal, Universidade Estadual de São Paulo, Jaboticabal, 2004.
SURFER for Windows. Realese 7.0. Contouring and 3D surface mapping for scientist's engineers. User's Guide. New York: Golden software, 1999. 619 p.

TSCHIEDEL, M.; FERREIRA, M. F. Introdução à agricultura de precisão: conceitos e vantagens. Ciência Rural, Santa Maria, v. 32, n. 1, p. 159-163, 2002.

VIEIRA, S. R. Geoestatística em estudos de variabilidade espacial do solo. In: NOVAIS, R. F.; ALVARES, V. H.; SCHAEFER, C. E. G. R. Tópicos em ciência do solo. Viçosa: Sociedade Brasileira de Ciência do Solo, 2000. v. 1, p. 1-53.

WARRICK, A. W.; NIELSEN, D. R. Spatial variability of soil physical properties in the field. In: HILLEL, D. (Ed.). Applications of soil physics. New York: Academic, 1980. cap. 2, p. 319-344.

WHITE, J. G.; WELCH, R. M.; NORVELL, W. A. Soil zinc map of the USA using geostatistics and geographic information system. Soil Science Society of America Journal, Madison, v. 61, n. 1, p. 185-194, 1997.

YANG, J. I.; MOSBY, D. E.; CASTEEL, S. W.; BLANCHAR, R. W. Microscale $\mathrm{pH}$ variability for assessing of phosphoric acid treatment in lead-contaminated soil. Soil Science, Baltimore, v. 66, n. 6, p. 374-381, 2001. 